\title{
Nucleic acid recognizing Toll-like receptors as therapeutic targets: a focus on autoimmunity and cancer
}

Philipp Yu

Institute for Immunology, Philipps-Universität-Marburg, Marburg, Germany
This article was published in the following Dove Press journal:

Journal of Receptor, Ligand and Channel Research

28 August 2009

Number of times this article has been viewed

\begin{abstract}
The "trinity" of nucleic acid recognizing Toll-like receptors (TLRs) is the focus of intense research efforts. Among the plethora of pattern recognition receptors the ones that recognize RNA and DNA always seemed Janus-faced in their potential - dangerous, but also promising. Therefore manipulating TLR3, TLR7 and TLR9, which recognize dsRNA-, ssRNAand CpG-containing DNA, respectively, is a very attractive goal, because increasing evidence shows that both suppression or enhancement of the immune system might thereby be achieved. In this review we try to illustrate the current knowledge of the endosomal nucleic acid recognizing TLRs. We then want to extend our view beyond the pathogen recognizing capacity to their role in autoimmunity and cancer. Significant progress has been made in our understanding of the structure of TLRs, signaling pathways and ligand identity. The question of if and how we can translate this into new specific immunosuppressive drugs or anti-cancer adjuvants is discussed.
\end{abstract}

Keywords: Toll-like receptors, immunosuppressive drugs, anti-cancer adjuvants

\section{A brief outline of Toll-like receptors in innate and acquired immunity}

About 25 years ago immunologists mainly focused on how antigen-specific immune responses were elicited. Model antigens, such as haptenated protein antigens, were used for experiments in order to reduce the complexity of the immune response. However, the immune response directed against pathogens in vivo is polyclonal and a complex mixture of innate and antigen specific effectors. Because immunologists embraced the revolutionary advances in molecular genetics they could quickly give answers to the question of how the adaptive immune system generates antibody or TCR diversity. ${ }^{1}$

However, this knowledge seemed incomplete, because the adaptive immune response against these antigens (highly purified and applied without adjuvant) was very weak compared to the responses generated by infections. ${ }^{2}$ While the importance of antigen-presenting cells (APCs) was already appreciated, it was not shown until later that both the quality of the antigen and the adjuvants used in an experimental immunization played a key role. For example, complete Freund's adjuvant (CFA), a mixture of mineral oil and dead mycobacteria, contains components that activate and direct the immune response by causing inflammation. Recently it was shown that Toll-like receptors (TLRs) recognize microbial products and cause an inflammatory cytokine response. This essential role of TLRs for the establishment of an adequate immune defense against numerous pathogens has been firmly established
Correspondence: Philipp Yu Institute for Immunology, PhilippsUniversität-Marburg, Hans Meerwein Str. 2, 35043 Marburg, Germany $\mathrm{Tel}+49-(0) 642 \mathrm{I}-28-66502$ Fax +49-(0) 642I-28-668I3 Email philipp.yu@staff.uni-marburg.de 
in recent years but had been postulated by the late Charles Janeway. He defined a long-standing dichotomy between adaptive and innate immunity, where flexible gene rearrangements define the specificity of antigen receptors of the adaptive immune system, in contrast to the germline encoded "fixed" pattern recognition receptors (PRRs) of the innate immune system. PRRs are able to recognize a molecular signature called a pathogen-associated molecular pattern (PAMP), which is expressed in the pathogen but not the host. In fact TLRs were the first family of receptors that fit perfectly with this view of the innate immune system. TLR are type I transmembrane proteins that are evolutionarily conserved between insects and vertebrates. In Drosophila, Toll was first identified as an essential molecule for dorsoventral patterning of the embryo and subsequently as a key molecule for the antifungal immune response in the adult animal. A homologous family of Toll receptors (termed TLRs) exists in vertebrates where 13 members (TLR1-13) are fundamental for the recognition of PAMPs. The family of TLRs recognizes various PAMPs of different pathogenic origin, such as bacteria, viruses, fungi or protozoan parasites (Table 1).

Since the general aspects of TLR structure, signaling and function have been discussed in excellent reviews ${ }^{3-6}$ we will focus on two aspects of TLRs that present valuable targets for pharmacological intervention in human disease. The TLR biology of autoimmunity and cancer is most interesting because it marks the two (pathological) extremes of what TLRs can do. By understanding the function of TLRs in self-recognition of autoantigens associated with autoimmune disease, we could not only cure these diseases, but we could also resolve an equally burning question: why do TLRs fail in recognition of cancer cells, although endogenous TLR ligands might be presented? Indeed an effective anti-tumor TLR response can be seen as a form of "beneficial autoreactivity".

The seminal point of mammalian TLR research was the discovery by Beutler et al that TLR4 was the long-sought LPS receptor. ${ }^{7}$ In a series of equally important findings it was shown over the last few years that this family of surface, eg, TLR4, and endosmomal, eg, TLR9, receptors are able to

Table I Overview of known Toll-like receptors (TLRs) in man and mouse and their ligands

\begin{tabular}{|c|c|c|c|}
\hline PRRs & Adapters & PAMPs/Activators & Source \\
\hline TLRI & MyD88, TIRAP & Triacyl lipopeptides & Bacteria \\
\hline \multirow[t]{8}{*}{ TLR2 } & MyD88, TIRAP & Diacyl lipopeptides & Mycoplasma \\
\hline & & Lipoteichonic acid & Bacteria \\
\hline & & Zymosan & Fungus \\
\hline & & PGN & Bacteria \\
\hline & & Lipoarabinomannan & Mycobacteria \\
\hline & & Porins & Bacteria (Neisseria) \\
\hline & & tGPI-mucin & Parasites (Trypanosoma) \\
\hline & & HA protein & Virus (measles virus) \\
\hline TLR3 & TRIF (TIR) & dsRNA & Virus \\
\hline \multirow[t]{5}{*}{ TLR4 } & MyD88, TIRAP, TRIF & LPS & Bacteria \\
\hline & TRAM & Envelope proteins & Virus (RSV, MMTV) \\
\hline & & HSP60 & Bacteria \\
\hline & & HMGBI & Host \\
\hline & & HSP60/70 & Host \\
\hline TLR5 & MyD88 & Flagellin & Bacteria \\
\hline TLR7 & MyD88 & ssRNA & RNA virus \\
\hline hTLR8 & MyD88 & ssRNA & RNA virus \\
\hline \multirow[t]{3}{*}{ TLR9 } & MyD88 & CpG DNA & Bacteria \\
\hline & & DNA & DNA virus \\
\hline & & Malaria hemozoin & Parasites \\
\hline TLRIO & $?$ & $?$ & $?$ \\
\hline \multirow[t]{2}{*}{ mTLRII (LRR-TIR) } & MyD88 & Not determined & Bacteria (uropathogenic bacteria) \\
\hline & & Profilin-like molecule & Parasites (Toxoplasma gondii) \\
\hline
\end{tabular}

Abbreviations: See page 26. 
recognize all kinds of PAMPs. This causes well-understood effects, like receptor dimerization and enhanced binding of the adaptor MyD88 (for clarity we have excluded the other adaptors TRAM/TRIF). Further important signaling events are the activation of IRAK family kinases 1 and 4 , TRAF3 and 6. This is followed by MAPK and IKKa/b activation, which differentially stimulates interferon alpha (IFN- $\alpha$ ) production via IRF7 and proinflammatory cytokines via NFkB, AP-1 and IRF-5. The activation of the proinflammatory cytokines, including IL-12, IL-6 and TNF- $\alpha$ and upregulation of costimulatory surface molecules like CD40, CD80, CD86 and MHCII on macrophages or DCs, ultimately leads to a Th1-dominated cellular immune response (Figure 1).

This explained how the addition of bacterial components as adjuvants, eg, Freund's adjuvant, enhances and activates a robust immune response. Indeed all pathogens contain (protein) antigens and a series of TLR ligands, which allow direct and indirect activation of adaptive immune cells. For example, infection with bacteria, which express LPS, ssRNA and non-methylated CpG DNA, engages TLR4, TLR7 and
TLR9, thereby indirectly activating a Th1 T cell response via, eg, DCs. On the other hand B cells also express these TLRs and, therefore, can be directly activated to proliferate and produce antibodies. So the humoral arm of the adaptive immunity is intricately connected with the innate immune system by expression of certain TLRs. This blurs the dichotomy between the innate and adaptive immune system and makes B cells in fact part of the innate immune response.

If, or how, T helper cells or Tregs directly react to certain TLR ligands like LPS, Pam-3-Cys or ssRNA is still a matter of debate. It is tempting to postulate that direct microbial Treg activation could abolish an inhibitory effect on Tregs during the activation phase of an immune response.

\section{Microbial recognition via TLR-like receptor mechanisms and their role in infection}

It is most interesting that the cosmos of TLR ligands includes viral, bacterial ligands and biocrystals (eg, hemozoin from malaria parasites), but also a fungal (yeast) cell

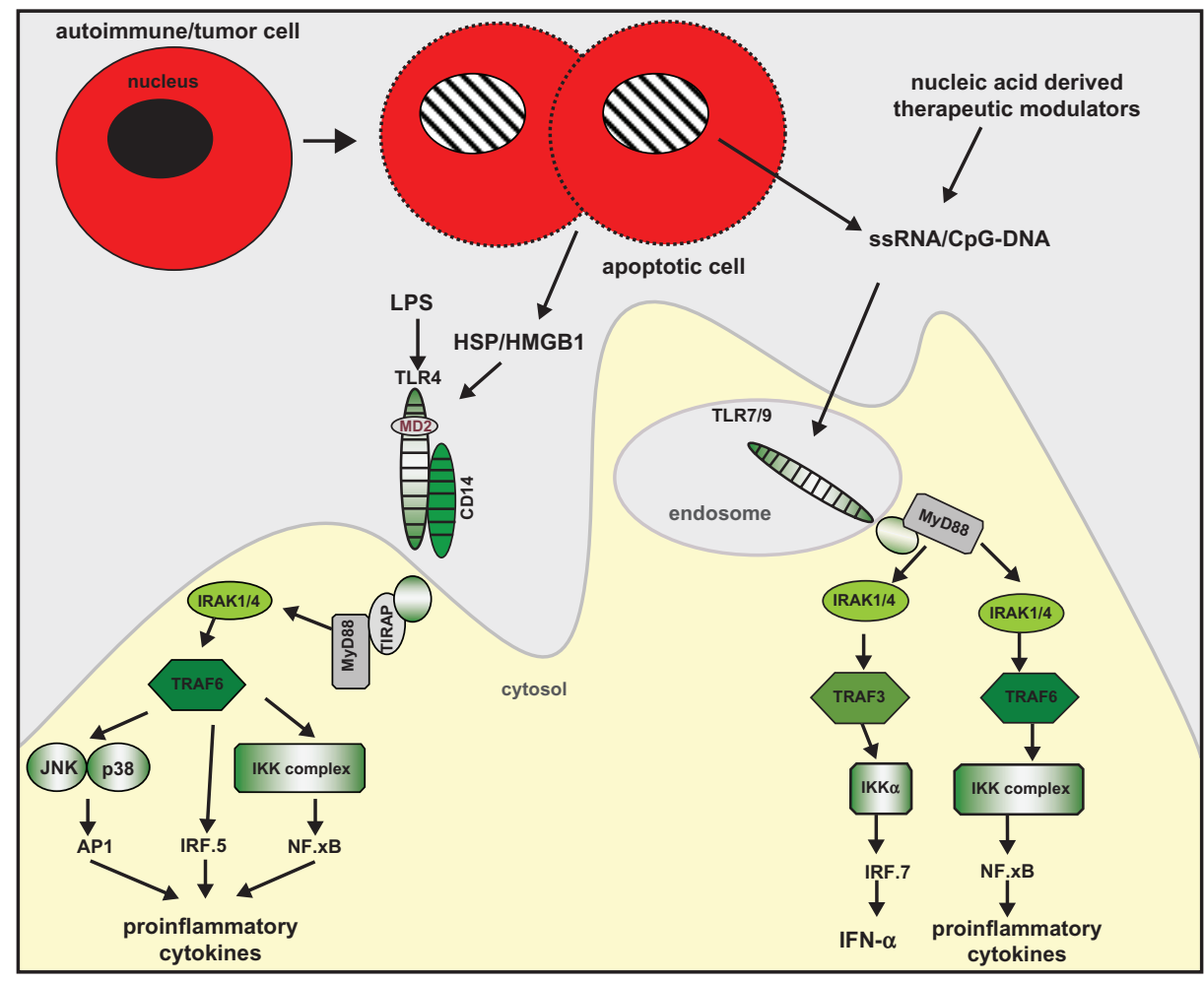

Figure I HMGBI (high-mobility group I), HSP (heat shock protein), RNA and DNA from autoimmune or tumor cells as the source for TLR-mediated immune activation. Both tumor surveillance and autoimmune activation can be mediated by TLRs. In addition nucleic acid-derived therapeutics have been developed that either block the undesirable effects of TLR engagement or activate an anti-tumor response through activation of downstream signaling and cytokine production. A simplified signal transduction is depicted, which results in the synthesis of the effector molecules, proinflammatory cytokines, and IFN- $\alpha$, TLR7 and TLR9 are sequestered in the endosome in order to avoid self-activation by RNA or DNA.

Abbreviations: See page 26. 
wall-derived protein-carbohydrate complex called zymosan (see Table 1). The recognition of ligands is dominated by molecules originating in viruses and bacteria. Identification of a recognition capacity for fungi and protozoan parasites by TLRs is also interesting, because they reveal a Janus-faced function of TLRs in the defense against fungi. Cell wall components of fungi such as zymosan and phospholipomannan are recognized by TLR2 and TLR4. The outcome of the recognition can vary depending on the receptor triggered. Since a Th1 immune response is important for clearance of fungal infection, the Th1-promoting activity of TLR4 enhances an effective immune response against fungi. Accordingly, TLR $4^{-/}$mice show increased susceptibility to disseminated Candida albicans infection. ${ }^{8}$ In contrast, TLR2 induces fewer Th1-inducing inflammatory cytokines and promotes, under certain conditions, production of IL-10, which can support Th2 immune responses and also downregulate immune responses. Thus, TLR2 $2^{-/-}$mice are more resistant to fungal infection. Infected TLR $2^{--}$mice show normal production of proinflammatory cytokines, but IL-10 secretion is severely impaired suggesting that $C$. albicans induces immunosuppression via IL-10. ${ }^{9}$ Aspergillus fumigatus, an opportunistic fungal pathogen, uses a similar mechanism for immune evasion. The non-infectious conidia are recognized by TLR 2 and TLR 4 whereas the more virulent hyphae are recognized only by TLR2, leading to immunosuppressive IL-10 production. However, for Cryptococcus neoformans, a pathogenic encapsulated yeast, both MyD88(-/-) and TLR2(-/-) animals showed decreased TNF- $\alpha$, IL-12p40 and/or IFN- $\gamma$ expression in various organs during infection. This again proves that the balance between suppressive and proinflammatory response decides pathogenic or protective outcome in fungal infections. ${ }^{10,11}$

This example shows that microbial recognition of complex pathogens results in the integration of different signals via antigen receptors, TLRs and other innate immune receptors.

It is interesting that a rather simple structure of leucinrich repeats (LRR) linked by a transmembrane region to a so-called Toll interleukin-1 receptor (TIR) domain can provide enough structural diversity to provide the binding capacity for the different TLR-ligands and complex signaling pathways. For two ligands the three-dimensional structure of ligand and extracellular TLR receptor binding domain has been solved: ${ }^{12}$ TLR4 with LPS and TLR3 with RNA. It is, indeed, conserved structural patterns that are recognized in diverse microbial molecules in the TLR family. The hydrophobic ligand of TLR4 interacts with internal protein pockets. ${ }^{13}$ In contrast, dsRNA, a hydrophilic ligand, interacts with the solvent-exposed surface of TLR $3 .{ }^{14}$ Binding induces dimerization of the ectodomains of the various TLRs, forming dimers that are strikingly similar in shape. In these " $m$ "-shaped complexes, the $\mathrm{C}$ termini of the extracellular domains of the TLRs converge in the middle. This observation suggests that dimerization of the extracellular domains forces the intracellular TIR domains to dimerize, which initiates signaling by recruiting intracellular adaptor proteins.

Although the list in Table 1 contains various molecules, no specific molecules from large parasites, eg, parasitic helminthic worms, have been identified as ligands for TLRs. The question remains if one of the remaining orphan TLRs could bind an antigen-derived from said group of pathogens, or alternatively if the recognition of these parasites depends on DNA or RNA recognition. A similarly possible explanation is that the Th1 dominated cellular immune response is not the best way to protect against worm parasites.

\section{TLR-like receptor deficiencies and TLR-mediated signaling in human infections}

The type of TLR ligand reveals what pathogens posed the strongest selective pressure on the immune system (Table 1) and forced it to develop these specific germ line-encoded TLRs. However, a more direct conclusion on the essential function of the individual TLRs can be obtained by analysis of TLR deficient mice. The laboratory of Shizuo Akira has been very successful in delineating both MyD88-dependent and -independent signaling pathways and the function of TLRs in general by using knockout mice. ${ }^{15}$ Recently basic mechanisms associated with IFN-a and proinflammatory signaling via TLRs were elucidated (see Figure 1).

Comparison of the murine and human signaling system revealed a large amount of homology. This might allow the quick transfer of knowledge gained in the mouse system to new therapeutic approaches in humans. Indeed, the analysis of patients with severe recurring infections, mainly during infancy, led to the discovery of primary genetic deficiencies of TLRs and associated signaling molecules and exciting new understandings of their function. From the seminal work under the direction of Jean-Laurant Casanova we learnt that what he called "experiments of nature", causing a genetic deficiency of either TLRs or associated molecules, leads to a rather restricted disease pattern of affected patients. ${ }^{16}$ For example, IRAK-4 deficient patients suffer from Grampositive pyogenic bacterial infections, leading to a death 
rate of $50 \%$ until adolescence. Then, however, the impaired immune system seems to be able to handle infections surprisingly well. For anti-viral immunity, it was suggested that TLR7-, TLR8- and TLR9-dependent induction of type I IFN is largely redundant in humans. However, the single immunodeficiency of TLR3 leads to susceptibility to HSV-1 in the central nervous system but a redundant immunity to most other viral infections. This can be indirectly concluded from serological data, which show that IRAK-4-, UNC-93B- and TLR3-impaired patients do have positive antibody tests against various DNA and RNA viruses How much redundancy in humans and mice is involved in TLR-mediated antiviral defense is a particularly interesting question for immunologists, and likewise for virologists and clinicians. ${ }^{17}$ Basic research has been very instrumental in obtaining the complementary knowledge in signaling of the TLRs. One current debate is the comparison of ssRNA-TLR7- and CpG DNA-TLR9-mediated signaling and cellular activation. Both can induce type I IFNs and different proinflammatory molecules, but a differential signaling capacity of TLR7 and TLR9 must be postulated. Recently it has been shown that a differential signaling capacity of TLR7 and TLR9 leads to differential thresholds in ligand mediate activation. This might lead to the selective usage of IRF7 by TLR7. ${ }^{18,19}$ However, further experiments are needed to solve finally the riddle of how selective signals are processed to activate cytokine-mediated immune activation in TLR7- or TLR9-associated disease states.

\section{Modulation of TLR response as a model for drug development opportunities in autoimmunity and cancer}

Only a handful of very rare cases of primary TLR-associated immundeficiencies have been described. However a surge of publications has addressed the question of how modulation of the TLRs or their downstream pathways is involved in autoimmune conditions, eg, inflammatory autoimmune disease like systemic lupus erythemadoes (SLE) or rheumatoid arthrits, which affect a substantial number of people.

We and others have been become interested in the role of nucleic acid-recognizing TLRs in particular TLR3, TLR7 and TLR9 in SLE-like autoimmune disease in order to lay the foundation for clinical trials in human disease. ${ }^{20}$ SLE is the prime candidate for an autoimmune disease of unknown etiology that could be linked to TLR modulation. Since pathomechanisms are complex it is not surprising that only imperfect animal models exist, which demands further preclinical studies to establish the molecular mechanisms in the human condition. Here we discuss data on TLRdeficient mice crossed with autoimmune prone mice, eg, with an upregulating point mutation in the phospholipase $\mathrm{C}$ $\gamma 2$ (Plcg2 ${ }^{\text {Ali5 }}$ ) molecule. SLE is characterized both by uncontrolled inflammatory response and mediated breakdown of B cell tolerance, resulting in anti-DNA autoantibody production and immune complex glomerulonephritis. For this multigenetic human disease of unknown etiology no single perfect animal model exists. For example, human SLE is associated with conserved Fas expression and lymphopenia, ${ }^{21}$ but the widely used murine model MRL-Fas ${ }^{\text {lpr }}$ displays a deficiency in Fas and lymphoproliferative disease..$^{22}$ Furthermore, data on TLR9 association with human SLE are conflicting and an influence of MyD88 or IRAK-4, two important signaling molecules of different TLRs, on human autoimmunity has been appreciated only recently. ${ }^{23}$ Different genetic murine models have been described that mimic one or more features of the disease. ${ }^{24}$ Models that have been used include DNAse II-deficient mice, ${ }^{25}$ Motheaten (SHP-1-deficient), MRL-Fas ${ }^{l p r}$, FcgRIIB-deficient ${ }^{26}$ and Plcg $2^{4 l i 5}$ mice. ${ }^{27}$ One can conclude that either a defect in apoptosis induction, clearance of potentially immunostimulatory cell debris or hyperactive BCR signaling may be an essential part of development of SLE-like autoimmunity. In order to examine the relation of these defects with the function of TLR9 in vivo, four different labs have used the approach of intercrossing TLR9-deficient mice with the latter three lupus models. ${ }^{28}$ One final example for a murine lupus model is the experimental induction of lupus-like symptoms by the transfer of alloreactive $\mathrm{T}$ cells. In this system a complex influence by TLR9 is executed which results in worsening of some aspects of autoimmunity while alleviating others. ${ }^{29}$

Although no current experimental data show that TLR9 has an influence on developmental processes, caution is necessary because the absence of TLR9 could indirectly affect lupus pathology in mouse systems. Therefore, careful comparison with non-TLR9-deficient models that use pharmacological inhibition of TLR9 signaling are mandatory for unbiased interpretation of TLR9 function in lupus. ${ }^{30}$ Furthermore, studies have been performed which demonstrate a crosstalk between TLR7/8 and TLR9, which recognize ssRNA and DNA, respectively, in the lpr SLE- model. ${ }^{31}$ We could corborrate these findings in multiple TLR-deficient autoimmune Plcg2 ${ }^{\text {Ali5 }}$ mice (unpublished data, P Yu). In summary, TLR9 signals differentially influence breakdown of B cell tolerance, anti-DNA autoantibody 
formation, antinuclear antibody (ANA)-specificity and, most importantly, the development of disease pathology. ${ }^{26}$

The mechanism by which Tlr9 signaling protects from inflammation in lupus like disease models is not yet understood. Type I IFN production might have a key role in SLE, ${ }^{32}$ because an IFN- $\alpha$ induced gene expression signature can be detected, and treatment with IFN- $\alpha$ can provoke SLElike symptoms in a small fraction of patients. ${ }^{33}$

In order to approach experimentally the role of type I IFNs in SLE-like autoimmunity, different labs have tested the effect of IFN- $\alpha$ treatment or genetic type I IFN receptor deficiency in the NZB and the MRL-Fas ${ }^{\mathrm{lpr}}$ model. While NZB mice are protected, MRL-Fas ${ }^{\mathrm{lpr}}$ mice display more severe autoimmunity, implying a protective effect of type I IFNs. ${ }^{34}$ One way to explain these contradicting results might be that MRL-Fas ${ }^{\mathrm{pr}}$ mice are a better model for the autoimmune lymphoproliferative syndrome (ALPS) caused by Fas mutations in humans. ${ }^{35,36}$ The anti-proliferative effect of IFN- $\alpha$ could be the mechanism that leads to a positive influence on autoimmunity in this model. Indeed, treatment of MRL-Fas ${ }^{\text {pr }}$ mice with IFN- $\beta$ prolonged survival and reduced severity of lupus-like disease. ${ }^{37}$ Despite the anti-proliferative effect, a general anti-inflammatory function of both type I IFNs and TLR9 was observed in an experimental colitis model and exacerbation of SLE-like symptoms in the respective genetic deficient mice. ${ }^{38,39}$ In support of these mouse data is a newly developed in vitro model of human PBMC stimulation, in which pretreatment with either type I IFN or TLR9 ligands inhibited the secretion of proinflammatory cytokines. ${ }^{40}$ The antagonism of pro- or anti-inflammatory action of the nucleic acid-recognizing TLRs in SLE-like murine disease also has a biochemical signaling aspect. It raises the question of how the endosomal ssRNA- and DNA-recognizing receptors,TLR7 and TLR9, respectively, execute their different functions at the signaling level, because known signaling molecules, eg, MyD88, Unc93b $1^{41}$ and IкB kinase-a ${ }^{19}$ are used by both TLRs. Recently, however, it was shown that the Unc93b1 "transporter" might bias the TLR response to nucleic acid toward DNA but against RNA-sensing. ${ }^{42}$ This very exciting finding might help to understand the balance between TLR7 and TLR9 transport to the endosome and its role in autoimmune disease. This very problem has been recently approached by the treatment of murine lupus prone models with different TLR7 and TLR9 inhibitors. The experiments did prove that there is a dominant ameliorating effect by inhibition of TLR7. ${ }^{43}$

It has not yet been analyzed if engagement of human TLR9 would have a similar protective effect in human SLE.
This is not only of academic interest but could give the ongoing clinical trials a new direction. In fact treatment with inhibitory ODNs specific for human TLR9 could aggravate SLE-symptoms, while engagement of TLR9 could ameliorate the disease in vivo. In addition the intracellular DNA sensors, ZBP-1/DAI and the inflammasome component ASC, ${ }^{44,45}$ which are different from TLR9, have been described recently and await further characterization with regard to SLE. Careful analysis will show if and how TLR9-specific drugs can be used in SLE therapy in the future. It is most interesting that the recent experimental focus has shifted from TLR $9,{ }^{46}$ as the primary pharmaceutical target for SLE inhibition, to TLR7. ${ }^{47}$ It was reported that lupus-prone mice deficient in TLR7 fail to generate antibodies to RNA-containing antigens such as Smith (Sm) Ag. Also, signs of clinical disease in lupus-prone TLR7-deficient mice were ameliorated and showed decreased lymphocyte activation, and decreased serum $\mathrm{IgG} .{ }^{48}$ These findings underscore an opposing inflammatory and regulatory role for TLR7 vs TLR9, despite similar signaling requirements in vivo. To add another layer of complexity it was shown that TLRs are not the only nucleic acid-recognizing receptors. We now know of two cytoplasmic RNA receptors, namely RIG-I and MDA-5. ${ }^{49}$

Indirect evidence for a cytoplasmic DNA receptor has been accumulating over recent years; however definite proof for the function of DAI or the other DNA receptor AIM2 $2^{50,51}$ in autoimmune activation by DNA has not been found. It will be a challenge to tease out the complex interactions between the different nucleic acid receptors and other genes associated with autoimmunity (eg, B cell activation genes). We can conclude that DNA and RNA do represent relevant autoantigens, but in addition CpG-containing DNA could be able to suppress SLE-like disease. The understanding of this differential function could lead to new therapeutics for human SLE.

In the ascending series of diseases, with an increasing number of affected patients, we have started with few patients with congenital defects in TLRs and then dealt with autoimmune patients with inflammatory autoimmune diseases (eg, SLE), which affects between 20 and 30 newly diagnosed patients per 100,000 persons. However, the largest group of patients that could potentially benefit from advances in TLR biology are cancer patients. The sheer number of cases and the limited options for therapy makes cancer a most important target for immunotherapy.

In particular, immune evasion mechanisms of cancer could be targets to boost defense against cancer. The complexity of the tumorigenic state ${ }^{52}$ and the limited success 
with adaptive anti-tumor therapy would call for aid from the innate immune system via TLR engagement. ${ }^{53}$

Before we go into details on how TLR-mediated mechanisms could be used against a tumor, we have to discuss how TLRs could have a negative function in the stimulation of tumorigenesis. ${ }^{54,55}$ The historical observation was that experimental transfer of cancer cells is enhanced by LPS administration. This led to increased invasiveness and angiogenesis. This is supported in the $\mathrm{Apc}^{\mathrm{Min} /+}$ model of carcinogenesis because when the mice are crossed with MyD88-deficient mice, the size of tumors and the incidence was decreased. It is not yet clear which specific TLRs are involved in the promotion of these sarcomas. Most recently it was shown that the extracellular matrix proteoglycan versican is produced by different carcinomas and acts as a TLR2 ligand which promotes metastatic progress in vivo. ${ }^{56}$ It is therefore possible that both tumor/metastasis-promoting and -inhibiting mechanisms are triggered by TLRs.

On the other hand, a positive correlation with bacterial infection and tumor remission was reported more than 100 years ago. It was W Coley who established a treatment with bacterial components in order to treat cancer. After the discovery of TLRs as a first line of defense against infection and potent primers of adaptive immune responses, it became clear that TLRs are the mediators of BCG-induced anti-tumor treatment. ${ }^{57}$ If one contemplates the question of how tumors induce a specific anti-tumor immune response at the innate immune level, it becomes obvious that if we look at a patient with an established tumor or metastasis, or a mouse that has developed an experimentally induced tumor, we examine the situation in which the immune response, either adaptive or innate, has failed to clear the tumor. This failure does not preclude the possibility that we can enhance a naturally weak or suboptimal innate immune response. Robert Schreiber has created the term immunoediting to suggest that a extended surveillance mechanism exists which determines the tumor qualities and the different stages of tumor immune system interactions. ${ }^{58}$ Here we will discuss what molecules are candidates for the innate anti-tumor response. We will focus on the question of if, and how, nucleic acid-recognizing receptors are involved in therapeutic anti-tumor defense or endogenous tumor surveillance.

TLRs involved in the recognition of structures unique to bacteria or fungi are expressed on the cell surface (TLR1, TLR2, TLR4, TLR5 and 6), whereas TLRs that recognize nucleic acids (TLR3, TLR7, TLR8 and TLR9) reside within intracellular compartments. In non-activated immune cells TLR9 is expressed in the endoplasmic reticulum (ER). Upon cellular activation, TLR9 traffics to endosomal and lysosomal compartments where it interacts with endocytosed $\mathrm{CpG}-\mathrm{DNA}$ at an acidic $\mathrm{pH}$, a condition that is thought to be necessary for DNA recognition. ${ }^{59}$ For TLRs located in the endosome, the nucleic acid has to enter these vesicles for activation. For certain viruses that enter the cell by receptor-mediated endocytosis (such as influenza virus) the encounter is mediated during enzymatic degradation of some virus particles in these vesicles. As nucleic acid is not unique to pathogens, the specificity of the nucleic acid-recognizing TLRs has been attributed to structural differences between eukaryotic and prokaryotic/viral nucleic acid. Accordingly, suppression of the CpG-DNA motif in eukaryotic DNA and frequent base modifications such as 5 'methyl-cytosine for DNA and 6'methyl-adenosine or 5'methyl-cytosine for RNA have been shown to be responsible for impaired recognition of eukaryotic nucleic acid by TLRs. ${ }^{45}$ In the context of tumor cell recognition it is interesting to note that tumors are believed to be hypomethylated. Therefore, a tumor in situ could have a substantially lower amount of $\mathrm{CpG}$ methylation, which could make it a more effective target for TLR9 recognition. This could then lead to IFN- $\alpha$ or TNF- $\alpha$ production, which could impair the tumor viability. In addition pDCs or macrophages that have taken up the tumor cells or the nucleic acid parts will be activated and express costimulatory molecules. Therefore, an efficient tumor antigen presentation, costimulatory help and anti-tumor cytokine response might be generated, which primes an adaptive anti-tumor immune response. However, structural differences among eukaryotic and prokaryotic DNA are presumably not the only mechanism to distinguish self from non-self DNA. Currently we are investigating whether an altered "tumor"-associated self-modification of DNA and RNA could have a place in immune activation. The situation is difficult because it is well known that apoptotic cells tend to be immune suppressive rather than activate the immune system. ${ }^{60}$ Furthermore, only in the pathological condition (eg, SLE) can eukaryotic DNA and RNA stimulate B cells and pDCs in a TLR-dependent manner. Being a multicausal disease, it is clear that a B cell-specific defect involving tolerance induction must be affected to cause immune activation by nucleic acids. Under natural conditions nucleic acids should be inert and not be recognized by the immune response. Whether an external inhibition of this level of tolerance could lead to better recognition of tumorassociated differences in nucleic acid, leading to inhibition of the tumor in vivo, needs to be studied in future. 
Apart from the modification of nucleic acid, the compartmentalization of the receptors (eg, expression in the endosome) is important to avoid recognition of selfDNA. Thus, the intracellular localization of the nucleic acid-recognizing TLRs provides a "safety mechanism" for avoiding the recognition of self-DNA that would occur if these TLRs were expressed at the cell surface.$^{61}$ The question of whether this "safety mechanism" is overridden when tumor cells die and RNA and DNA are released, taken up by APCs and ultimately transported to the endosomal TLRs is important when we suggest a role for TLR3, TLR7/8 and TLR9 in tumor surveillance. It is clear that "danger signals" from tumor cells represent a difficult task for the immune system because transformation of a body cell might not produce a proper danger signal to break self-tolerance and activate an efficient $\mathrm{T}$ cell or antibody response against tumor antigens. ${ }^{62}$ As can be seen in Table 1, a restricted number of self-TLR ligands have been described and only for few molecules has a direct role in anti-tumor immunity been assigned (eg, HMGB1). For HMGB1 it was demonstrated that chemotherapy-induced cell-death causes the release of HMGB1, which binds to TLR4 and activates an anti-tumor T cell response. ${ }^{63}$

For the nucleic acid-recognizing TLRs, two pathways for a anti-cancer mechanism have been discussed. First the described immune-activating effect of, eg, TLR7, for which it has already been proven that a small-molecule ligand has a two-fold benefical effect on certain skin cancers. Local activation by imiquimod is efficient in defense against genital warts caused by papilloma virus. At the same time basal cell carcinoma can also be treated with imiquimod, implying both an anti-viral and anti-tumor effect. ${ }^{64}$ It can be concluded that recognition of microbial RNA and DNA could indeed have a dual function in tumor defense. First, a variety of microbial pathogens associated with tumor transformation might be repelled by TLR-mediated innate immunity. For example, Epstein-Barr virus, hepatitis B or C, or Helicobacter pylori all express a variety of TLR ligands, including RNA and CpG DNA, which should allow activation of TLRs.

In fact, understanding the quality of modifications of RNA or DNA is becoming an increasingly important field to further our understanding of nucleic acids as danger signals. In this context it needs to be analyzed how RNA or DNA from dying or apoptotic tumor cells, and virus-infected cells, are modified to be recognized by the immune system. It is an equally important topic to identify mechanisms by which genomic DNA or RNA from normal cells is tolerated.
It seems that the primary effect of TLR9 ligands in cancer therapy is to act as adjuvants through upregulation of costimulatory molecules, thereby breaking self-tolerance against tumor antigens. It was first demonstrated that nonmethylated CpG motifs of DNA are the physiological ligand. Recently the structural specificity was analyzed in more detail in order to understand if non-CpG DNA, in the form of genomic DNA, could be a proper TLR9 ligand. Because this question is not definitively answered yet, both a role for lymphocyte activation in SLE and in natural immunity against tumors needs further experimental verification. While a role in tumor surveillance is still the subject of controversial discussion, the therapeutic application of activating TLR9 ligands in tumor therapy is progressing in clinical trials.

\section{Conclusions}

Existing knowledge of TLRs, both in general and in nucleic acid-driven immune responses of the innate immune system, puts us in a very promising position. We can use structural data, information about mouse TLR biology and what we know about human TLRs to build a strong framework for the development of RNA- and DNA-based rational therapies for autoimmunity and cancer.

\section{Abbreviations}

PGN, proteoglycan; RSV, respiratory-syncytial virus; MMTV, mouse mammary tumor virus; LPS, lipopolysaccharide; HMGB1, high mobility group 1; DAI, DNA-dependent activator of IFN-regulatory factors; BCG, Bacille Calmette Guerin (attenuated Mycobacterium bovis); ODN, oligodeoxy-nucleotide; PRR, pattern recognition receptor; PAMP, pathogen-associated molecular pattern.

\section{Acknowledgments and disclosures}

We thank Svetlana Hamm for help with the figures. Stephanie Beil for technical assistance and Allison Groseth for help with the manuscript. The author has no conflicts of interest to disclose.

\section{References}

1. Early $\mathrm{P}$, et al. An immunoglobulin heavy chain variable region gene is generated from three segments of DNA: VH, D and JH. Cell. 1980;19:981-992.

2. Lambrecht BN, et al. Mechanism of action of clinically approved adjuvants. Curr Opin Immunol. 2009;21:23-29.

3. Hoebe K, Beutler B. Forward genetic analysis of TLR-signaling pathways: an evaluation. Adv Drug Deliv Rev. 2008;60:824-829.

4. Wagner H. Endogenous TLR ligands and autoimmunity. Adv Immunol. 2006;91:159-173. 
5. Barrat FJ, Coffman RL. Development of TLR inhibitors for the treatment of autoimmune diseases. Immunol Rev. 2008;223:271-283.

6. Kawai T, Akira S. Toll-like receptor and RIG-I-like receptor signaling. Ann N Y Acad Sci. 2008;1143:1-20.

7. Beutler B. Microbe sensing, positive feedback loops, and the pathogenesis of inflammatory diseases. Immunol Rev. 2009;227: 248-263.

8. Netea MG, et al. An integrated model of the recognition of Candida albicans by the innate immune system. Nat Rev Microbiol. 2008; 6:67-78.

9. Netea MG, et al. Toll-like receptor 2 suppresses immunity against Candida albicans through induction of IL-10 and regulatory T cells. J Immunol. 2004;172:3712-3718.

10. Yauch LE, et al. Involvement of CD14, toll-like receptors 2 and 4, and MyD88 in the host response to the fungal pathogen Cryptococcus neoformans in vivo. Infect Immun. 2004;72:5373-5382.

11. Biondo C, et al. MyD88 and TLR2, but not TLR4, are required for host defense against Cryptococcus neoformans. Eur J Immunol. 2005;35:870-878.

12. Jin MS, Lee JO. Structures of the toll-like receptor family and its ligand complexes. Immunity. 2008;29:182-191.

13. Park BS, et al. The structural basis of lipopolysaccharide recognition by the TLR4-MD-2 complex. Nature. In press 2009.

14. Liu L, et al. Structural basis of toll-like receptor 3 signaling with doublestranded RNA. Science. 2008;320:379-381.

15. Kawai T, Akira $S$. The roles of TLRs, RLRs and NLRs in pathogen recognition. Int Immunol. 2009;21:317-337.

16. Zhang SY, et al. Human Toll-like receptor-dependent induction of interferons in protective immunity to viruses. Immunol Rev. 2007; 220:225-236.

17. Bustamante $\mathrm{J}$, et al. Novel primary immunodeficiencies revealed by the investigation of paediatric infectious diseases. Curr Opin Immunol. 2008;20:39-48.

18. Hacker $\mathrm{H}$, et al. Specificity in Toll-like receptor signalling through distinct effector functions of TRAF3 and TRAF6. Nature. 2006;439: 204-207.

19. Hoshino K, et al. IkappaB kinase-alpha is critical for interferonalpha production induced by Toll-like receptors 7 and 9. Nature 2006;440:949-953.

20. Lafyatis R, Marshak-Rothstein A. Toll-like receptors and innate immune responses in systemic lupus erythematosus. Arthritis Res Ther. 2007;9:222.

21. Rivero SJ, et al. Lymphopenia in systemic lupus erythematosus. Clinical, diagnostic, and prognostic significance. Arthritis Rheum. 1978;21:295-305.

22. Rieux-Laucat F, et al. Autoimmune lymphoproliferative syndromes: genetic defects of apoptosis pathways. Cell Death Differ. 2003;10: 124-133.

23. Isnardi I. et al. IRAK-4- and MyD88-dependent pathways are essential for the removal of developing autoreactive B cells in humans. Immunity. 2008;29:746-757.

24. Kono DH, Theofilopoulos AN. Genetics of SLE in mice. Springer Semin Immunopathol. 2006;28:83-96.

25. Okabe Y, et al. Toll-like receptor-independent gene induction program activated by mammalian DNA escaped from apoptotic DNA degradation. J Exp Med. 2005;202:1333-1339.

26. Ehlers M, et al. TLR9/MyD88 signaling is required for class switching to pathogenic IgG2a and $2 \mathrm{~b}$ autoantibodies in SLE. $J$ Exp Med 2006;203:553-561.

27. Yu P, et al. Autoimmunity and inflammation due to a gain-of-function mutation in phospholipase $\mathrm{C}$ gamma 2 that specifically increases external Ca2+ entry. Immunity. 2005;22;451-465.

28. Marshak-Rothstein A. Toll-like receptors in systemic autoimmune disease. Nat Rev Immunol. 2006;6:823-835.

29. Ma Z, et al. Modulation of autoimmunity by TLR9 in the chronic graft-vs-host model of systemic lupus erythematosus. $J$ Immunol. 2006;177:7444-7450.
30. Pawar RD, et al. Ligands to nucleic acid-specific Toll-like receptors and the onset of lupus nephritis. J Am Soc Nephrol. 2006.

31. Christensen SR, et al. Toll-like receptor 7 and TLR9 dictate autoantibody specificity and have opposing inflammatory and regulatory roles in a murine model of lupus. Immunity. 2006;25:417-428.

32. Banchereau J, Pascual V. Type I interferon in systemic lupus erythematosus and other autoimmune diseases. Immunity. 2006;25: 383-392.

33. Ronnblom L, Alm GV. Systemic lupus erythematosus and the type I interferon system. Arthritis Res Ther. 2003;5:68-75.

34. Hron JD, Peng SL. Type I IFN protects against murine lupus. J Immunol. 2004;173:2134-2142.

35. Infante AJ, et al. The clinical spectrum in a large kindred with autoimmune lymphoproliferative syndrome caused by a Fas mutation that impairs lymphocyte apoptosis. J Pediatr.1998;133:629-633.

36. Del-Rey M, et al. A homozygous Fas ligand gene mutation in a patient causes a new type of autoimmune lymphoproliferative syndrome. Blood. 2006;108:1306-1312.

37. Schwarting A, et al. Interferon-beta: a therapeutic for autoimmune lupus in MRL-Faslpr mice. J Am Soc Nephrol. 2005;16:3264-3272.

38. Lau CM, et al. RNA-associated autoantigens activate B cells by combined B cell antigen receptor/Toll-like receptor 7 engagement. $J$ Exp Med. 2005;202:1171-1177.

39. Pisitkun $P$, et al. Autoreactive B cell responses to RNA-related antigens due to TLR7 gene duplication. Science. 2006;312:1669-1672.

40. Meyers JA, et al. Blockade of TLR9 agonist-induced type I interferons promotes inflammatory cytokine IFN-gamma and IL-17 secretion by activated human PBMC. Cytokine. 2006;35:235-246.

41. Tabeta K, et al. The Unc93b1 mutation 3d disrupts exogenous antigen presentation and signaling via Toll-like receptors 3, 7 and 9 . Nat Immunol. 2006;7:156-164.

42. Fukui R, et al. Unc93B1 biases Toll-like receptor responses to nucleic acid in dendritic cells toward DNA- but against RNA-sensing. $J$ Exp Med. 2009;206:1339-1350.

43. Barrat FJ, et al. Treatment of lupus-prone mice with a dual inhibitor of TLR7 and TLR9 leads to reduction of autoantibody production and amelioration of disease symptoms. Eur J Immunol. 2007;37:3582-3586.

44. Takeshita F, Ishii KJ. Intracellular DNA sensors in immunity. Curr Opin Immunol. 2008;20:383-388.

45. Muruve DA, et al. The inflammasome recognizes cytosolic microbial and host DNA and triggers an innate immune response. Nature. 2008;452:103-107.

46. Vollmer J, Krieg AM. Immunotherapeutic applications of $\mathrm{CpG}$ oligodeoxynucleotide TLR9 agonists. Adv Drug Deliv Rev. 2009; 61:195-204.

47. Tluk S, et al. Sequences derived from self-RNA containing certain natural modifications act as suppressors of RNA-mediated inflammatory immune responses. Int Immunol. 2009.

48. Herlands RA, et al. T cell-independent and toll-like receptordependent antigen-driven activation of autoreactive B cells. Immunity. 2008:29:249-260

49. Schlee M, et al. Approaching the RNA ligand for RIG-I? Immunol Rev. 2009;227:66-74.

50. Hornung V, et al. AIM2 recognizes cytosolic dsDNA and forms a caspase-1-activating inflammasome with ASC. Nature. 2009;458: 514-518.

51. Fernandes-Alnemri T, et al. AIM2 activates the inflammasome and cell death in response to cytoplasmic DNA. Nature. 2009;458:509-513.

52. Luo J, et al. Principles of cancer therapy: oncogene and non-oncogene addiction. Cell. 2009;136:823-837.

53. Dougan M, Dranoff G. Immune therapy for cancer. Annu Rev Immunol. 2009;27:83-117.

54. Rakoff-Nahoum S, Medzhitov R. Toll-like receptors and cancer. Nat Rev Cancer. 2009;9:57-63.

55. Bui JD, Schreiber RD. Cancer immunosurveillance, immunoediting and inflammation: independent or interdependent processes? Curr Opin Immunol. 2007;19:203-208. 
56. Kim S, et al. Carcinoma-produced factors activate myeloid cells through TLR2 to stimulate metastasis. Nature. 2009;457:102-106.

57. Tsuji S, et al. Maturation of human dendritic cells by cell wall skeleton of Mycobacterium bovis bacillus Calmette-Guerin: involvement of toll-like receptors. Infect Immun. 2000;68:6883-6890.

58. Dunn GP, et al. The immunobiology of cancer immunosurveillance and immunoediting. Immunity. 2004;21:137-148.

59. Kim YM, et al. UNC93B1 delivers nucleotide-sensing toll-like receptors to endolysosomes. Nature. 2008;452:234-238.

60. Tesniere A, et al. Immunogenic cancer cell death: a key-lock paradigm. Curr Opin Immunol. 2008;20:504-511.
61. Barton GM, et al. Intracellular localization of Toll-like receptor 9 prevents recognition of self DNA but facilitates access to viral DNA. Nat Immunol. 2006;7:49-56.

62. Jeannin P, et al. Pattern recognition receptors in the immune response against dying cells. Curr Opin Immunol. 2008;20:530-537.

63. Apetoh L, et al. The interaction between HMGB1 and TLR4 dictates the outcome of anticancer chemotherapy and radiotherapy. Immunol Rev. 2007;220:47-59.

64. Broomfield SA, et al. Locally administered TLR7 agonists drive systemic antitumor immune responses that are enhanced by antiCD40 immunotherapy. J Immunol. 2009;182:5217-5224.

\section{Publish your work in this journal}

The Journal of Receptor, Ligand and Channel Research is an international, peer-reviewed, open access, online journal. The journal welcomes laboratory and clinical findings in the fields of biological receptors, ligands, channel and signal transduction research including: receptors and signalling; ligands; transporters, pores and channels; binding and activation; receptor regulation; role of receptors in diseases and their treatment; molecular basis of membrane structure and functions; molecular models of membranes. The manuscript management system is completely online and includes a very quick and fair peer-review system. Visit http://www.dovepress.com/ testimonials.php to read real quotes from published authors.

Submit your manuscript here: http://www.dovepress.com/journal-of-receptor-ligand-and-channel-research-journal 